\title{
Current Management of Patients with RPE65 Mutation-Associated Inherited Retinal Degenerations in Europe: Results of a Multinational Survey by the European Vision Institute Clinical Research Network
}

\author{
Birgit Lorenz $^{\mathrm{a}, \mathrm{b}}$ Joana Tavares ${ }^{\mathrm{c}}$ L. Ingeborgh van den Born ${ }^{\mathrm{d}}$ \\ João P. Marques ${ }^{e, f, g}$ Hendrik P.N. Schollh, ${ }^{h, j}$ The EVICR.net Group

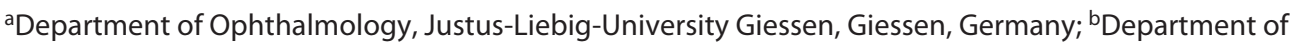 \\ Ophthalmology, University Hospital Bonn, Bonn, Germany; ' Association for Innovation and Biomedical Research

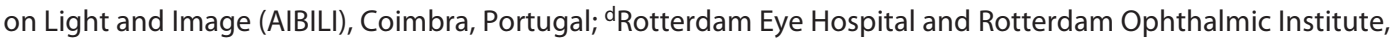 \\ Rotterdam, The Netherlands; ${ }^{2}$ Center for Clinical Trials, Association for Innovation and Biomedical Research on \\ Light and Image (AIBILI), Coimbra, Portugal; ' Department of Ophthalmology, Centro Hospitalar e Universitário \\ de Coimbra (CHUC), Coimbra, Portugal; '9Faculty of Medicine, University of Coimbra (FMUC), Coimbra, Portugal; \\ hInstitute of Molecular and Clinical Ophthalmology Basel (IOB), Basel, Switzerland; iDepartment of Ophthalmology, \\ University of Basel, Basel, Switzerland; jWilmer Eye Institute, Johns Hopkins University, Baltimore, MD, USA
}

\section{Keywords}

Inherited retinal degenerations $\cdot$ RPE65 - Management . Europe · European Vision Institute Clinical Research Network clinical centers

\begin{abstract}
Purpose: The first ocular gene augmentation therapy, voretigene neparvovec (VN) (Luxturna ${ }^{\circledR}$ ), has been approved for clinical use in an increasing number of countries (FDA USA 2017, EMA Europe 2018, MoHAP United Arab Emirates 2019, SFDA Saudi Arabia 2019, Swiss Medic Switzerland 2020, TGA Australia 2020, BFR Brazil 2020). Among the EVICR.net clinical centers, we conducted the first multinational survey to understand distribution, diagnostic work-up, and manage-
\end{abstract}

ment of inherited retinal degeneration (IRD) cases in Europe with a special focus on RPE65 mutation-associated IRDs. Methods: An electronic survey questionnaire including 35 questions specifically addressing RPE65 mutation-associated IRDs was developed and sent to the 101 EVICR.net clinical centers. Results: The overall response rate was 49\%. Fortytwo centers see IRD patients, and 22/42 follow patients with confirmed biallelic RPE65 mutations. Fifteen of the 22 centers (68\%) and 3/22 (14\%) follow 1-5 and 6-10 patients with homozygous RPE65 mutations, respectively. Additionally, $15 / 22(68 \%)$ and $3 / 22$ (14\%) follow $1-5$ and $>20$ patients with compound heterozygous RPE65 mutations, respectively. Fif-

Birgit Lorenz and Joana Tavares contributed equally. karger@karger.com www.karger.com/ore

Karger $\stackrel{\text { ' }}{5}$

BOPEN ACCESS
(C) 2021 The Author(s)

Published by S. Karger AG, Basel

This is an Open Access article licensed under the Creative Commons Attribution-NonCommercial-4.0 International License (CC BY-NC) (http://www.karger.com/Services/OpenAccessLicense), applicable to the online version of the article only. Usage and distribution for commercial purposes requires written permission.
Correspondence to:

Birgit Lorenz, birgit.lorenz@ uniklinikum-giessen.de 
ty-nine percent of mutations were ACMG Class 4 and 5 (at least 1 allele), $82.8 \%$ reported previously and $17.2 \%$ novel. Referral diagnoses (the mean per center) were Leber congenital amaurosis (38.2\%), early-onset severe retinal degeneration $(16.8 \%)$, rod-cone-dystrophy/retinitis pigmentosa (RP) (28.1\%), and unclassified visual impairment (17.0\%). Twenty-five percent of the centers changed the referral diagnosis in $>47.5 \%$ of cases; $32 \%$ follow a specific referral process for RPE65 mutation-associated IRD patients. Annual follow-up visits are done in $55 \%$ of the centers and biannual visits in $23 \%$. In $32 \%$, other centers also follow the patients. Kinetic perimetry is done in $82 \%$, static perimetry in $45 \%$, and microperimetry in $18 \%$ of the centers. Full-field light stimulus threshold testing with blue and red stimuli to quantify the rod and cone function is used in $6 / 22$ centers (27\%). A mobility course is available in one center (5\%). Conclusion: This first multinational survey on management of patients with RPE65 mutation-associated IRDs in Europe shows that about half of the responding EVICR.net centers have such patients under care. There is heterogeneity in diagnoses and management practices. At the start of clinical practice experience with $\mathrm{VN}$, these data provide a useful baseline and highlight the need for consensus/guidelines to inform standard of care in this new era of gene therapy.

(C) 2021 The Author(s)

Published by S. Karger AG, Basel

\section{Introduction}

RPE65 mutation-associated inherited retinal degenerations (IRDs) are of great interest as an approved therapy is now available in an increasing number of countries worldwide (FDA USA 2017, EMA Europe 2018, MoHAP United Arab Emirates 2019, SFDA Saudi Arabia 2019, Swiss Medic Switzerland 2020, TGA Australia 2020, BFR Brazil 2020). Cost-effectiveness of this therapy for the national health-care system is actually discussed in a number of articles [1-5]. The gene was identified by 2 groups independently in 1997 [6, 7], and while Marlhens et al. [6] reported mutations in patients with autosomal recessive Leber congenital amaurosis, $\mathrm{Gu}$ et al. [7] had found mutations in patients with autosomal recessive childhood-onset severe retinal dystrophy. Recent reports have described a yet wider range of diagnoses [8-12], which has important implications as to the population that should be screened for biallelic mutations in RPE65.

The aim of this study is to specifically report and analyze in detail diagnosis and management of RPE65 mutation-associated IRDs $[8,13,14]$ across Europe. The

Survey on RPE65 Mutation-Associated IRDs Conducted in Europe
EVICR.net retinal dystrophies Expert Committee conducted an electronic survey questionnaire aimed to understand the current management of IRD cases across the 101 EVICR.net clinical centers [15]. We complemented the general survey by 35 additional questions specifically related to RPE65 mutation-associated IRDs. The survey gives important insights into the epidemiology of RPE65 mutation-associated IRDs and the range of clinical diagnoses in a number of patients eventually amenable to therapy. Increased awareness for the range of first diagnoses of RPE65 mutation-associated IRDs will help identify more patients that might benefit from therapy. It will also further improve our understanding of the disease course in relation to the underlying mutations, since some exceptional patients have a slower progression than others [11]. These observations are important to decide on the best time point in the disease course for gene augmentation therapy, since the optimal window for successful treatment remains yet to be identified. The typically used readout parameters are still not optimal to identify cells that can be salvaged by the intervention [16]. In treated 5- to 6-year-old dogs with biallelic RPE65 mutations, during an observation period of $4-5$ years, areas with $>63 \%$ of retained photoreceptors at the time of treatment showed robust retention of photoreceptors, whereas areas with less retained photoreceptors showed continuous degeneration similar to what had been reported earlier [17]. An unexpected finding was that both treated and untreated regions in study eyes tended to have less degeneration compared to matched locations in untreated control eyes. Although animal data cannot be strictly compared to the human phenotype, the findings do open new aspects on when and where to treat and underline the need of identifying as many patients with RPE65 mutation-associated IRDs as possible to answer such questions. RPE65 mutation-associated IRDs are a rare form of IRD with an estimated prevalence of about 1 in 300,000 [1, 18]. Given the overall population of Europe of $747,700,446$ as of Friday, August 21, 2020, based on the latest United Nations estimates, we can expect about 2,500 patients with biallelic mutations in RPE65 in Europe. As many of the patients are blind by the end of the third or fourth decade of life $[8,12,19]$, the number of patients who might benefit from gene supplementation therapy is likely much lower. Early diagnosis is important to investigate treatment potential at early stages of the disease, aiming not only to improve and preserve the rod function, but also to improve and maintain the cone function. 
Table 1. Estimate number of IRD patients with confirmed homozygous and compound heterozygous mutations in RPE65

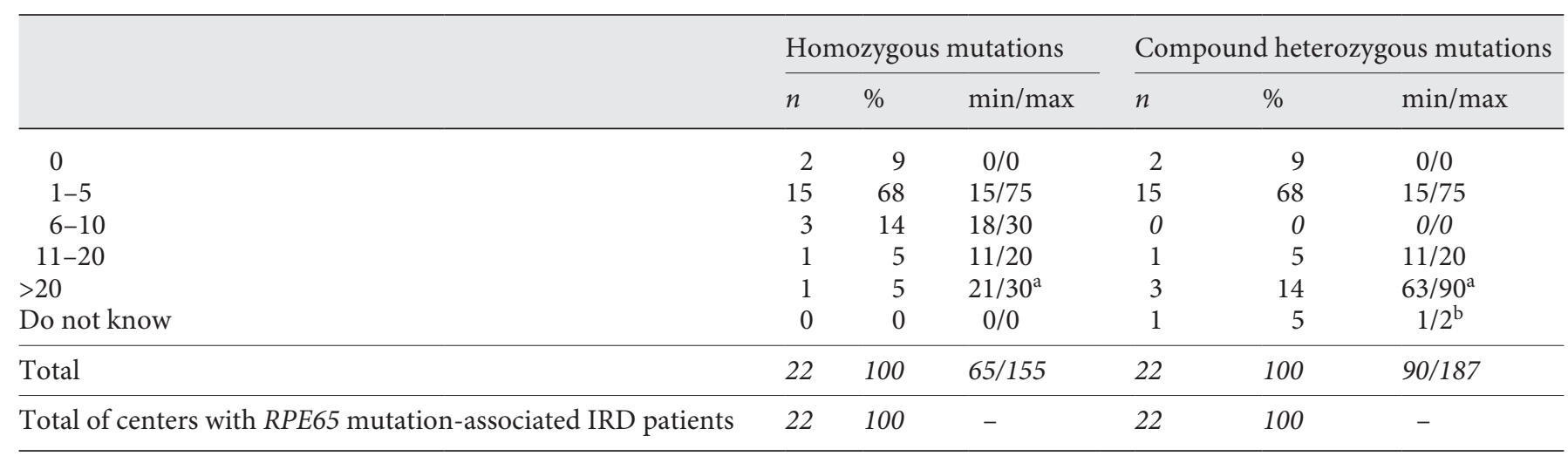

$N$ refers to the number of centers. IRD, inherited retinal degeneration. ${ }^{a}$ Estimated number assuming 30 patients as maximum for centers with $>20$ patients. ${ }^{b}$ Estimated number assuming 2 patients as maximum for centers answering do not know.

\section{Materials and Methods}

\section{Study Design}

We carried out a cross-sectional study among ophthalmic clinical centers with an EVICR.net membership from 14 European countries (CH, DE, FR, IT, UK, SP, DK, AU, BE, GR, IL, NL, PT, and SK) and Israel. In May 2019, all EVICR.net clinical centers were invited by e-mail to complete the online questionnaire. This invitation was sent to the responsible person of the clinical center and also to its representative for the EVICR.net Retinal Dystrophies Scientific Section; however, no restrictions were imposed to participate in the survey (shared via public link). Therefore, any member of the clinical center staff (e.g., medical retina ophthalmologist, general ophthalmologist, pediatric ophthalmologist, and other) could have replied to the survey on their behalf. Only one reply per clinical center was considered.

A reminder was sent to the non-repliers after 2 weeks, the deadline was extended for 2 more weeks, and new reminders were sent on week 3 and week 4 (2 days before deadline). Several strategies were applied to increase the response rate, namely, follow-up contact, hard copy of the questionnaire, personalized e-mails, and giving a deadline.

\section{Questionnaire}

An IRD Expert Committee developed the IRD survey questionnaire. This Committee members were Birgit Lorenz, MD PhD, Germany (Scientific Coordinator); Hendrik Scholl, MD PhD, Switzerland; Isabelle Audo, MD PhD, France; Ingeborgh van den Born, MD PhD, the Netherlands; and João Pedro Marques, MD, Portugal.

The questionnaire was divided in 5 sections: section 1: IRD demographics, section 2: local setting, section 3: IRD genetic testing and counselling, section 4: involvement in clinical trials, and section 5: RPE65 mutation-associated IRDs. The results from sections 1 to 4 have been reported separately [15]. Here, we present the results from section 5 . Section 5 comprised 35 questions that followed a conditional branching (see online suppl. material - online questionnaire; see www.karger.com/doi/10.1159/000515688 for all online suppl. material). The questionnaire was designed to

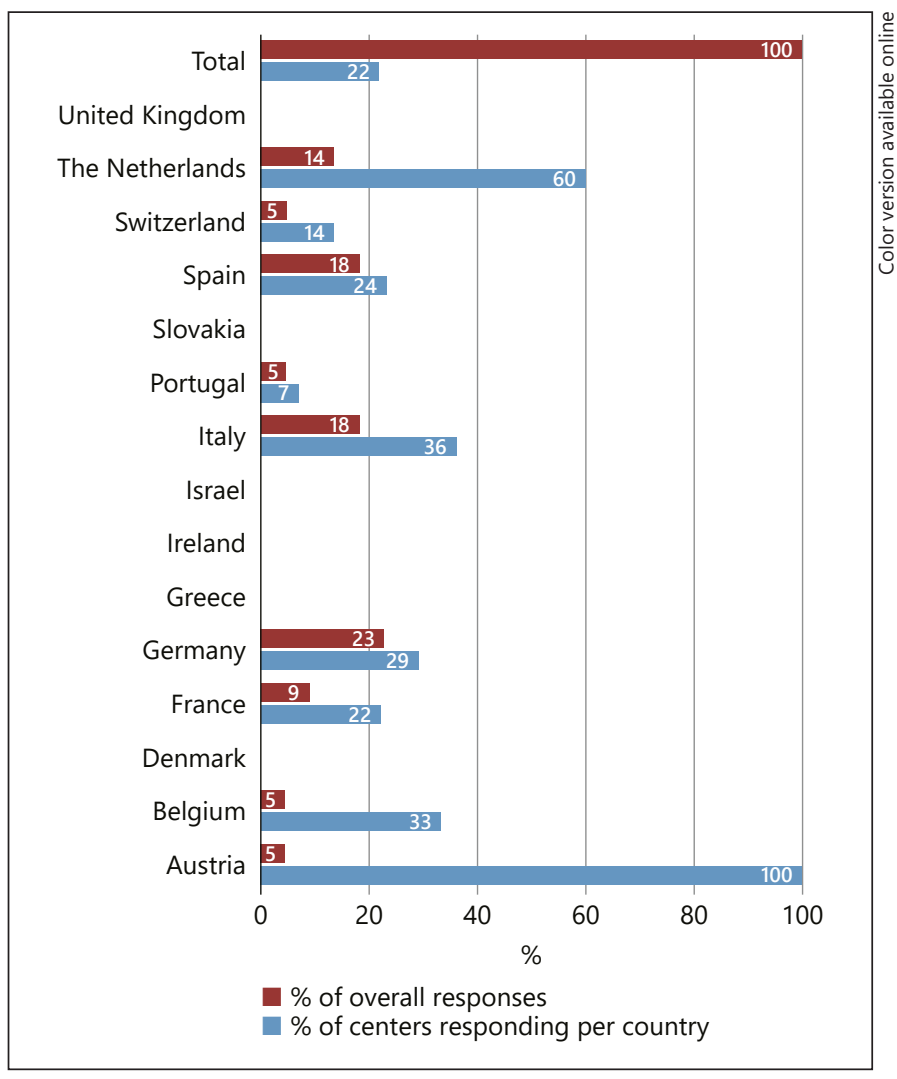

Fig. 1. Centers that reported to have IRD patients with confirmed biallelic mutations in RPE65 identified in their centers by country. IRD, inherited retinal degeneration.

have mostly multiple-choice questions and single choice questions (closed-ended items), in which the options represent a range of values, which means that only estimates were requested. Due to the low number of patients with RPE65 biallelic mutations, we decided 
Table 2. Estimate number of IRD patients with confirmed biallelic mutations in RPE65 distributed by country

\begin{tabular}{|c|c|c|c|c|}
\hline Country & $N$ & $\begin{array}{l}\text { Total centers at } \\
\text { section } 5 \text { per country }\end{array}$ & $\begin{array}{l}\% \text { per } \\
\text { country }\end{array}$ & $\operatorname{Min} / \max$ \\
\hline \multicolumn{5}{|c|}{ Homozygous mutations in RPE65 } \\
\hline Total & 22 & 22 & - & $65 / 155$ \\
\hline Austria & 1 & 1 & 100 & $0 / 0$ \\
\hline Germany & 1 & 5 & 20 & $0 / 0$ \\
\hline Total & 2 & - & - & $0 / 0$ \\
\hline \multicolumn{5}{|l|}{$1-5$} \\
\hline Belgium & 1 & 1 & 100 & $1 / 5$ \\
\hline France & 2 & 2 & 100 & $2 / 10$ \\
\hline Germany & 2 & 5 & 40 & $2 / 10$ \\
\hline Italy & 3 & 4 & 75 & $3 / 15$ \\
\hline Portugal & 1 & 1 & 100 & $1 / 5$ \\
\hline Spain & 3 & 4 & 75 & $3 / 15$ \\
\hline Switzerland & 1 & 1 & 100 & $1 / 5$ \\
\hline The Netherlands & 2 & 3 & 67 & $2 / 10$ \\
\hline Total & 15 & - & - & $15 / 75$ \\
\hline \multicolumn{5}{|l|}{$6-10$} \\
\hline Germany & 2 & 5 & 40 & $12 / 20$ \\
\hline Italy & 1 & 4 & 25 & $6 / 10$ \\
\hline Total & 3 & - & - & $18 / 30$ \\
\hline \multicolumn{5}{|l|}{$11-20$} \\
\hline The Netherlands & 1 & 3 & 33 & $11 / 20$ \\
\hline Total & 1 & - & - & $11 / 20$ \\
\hline \multicolumn{5}{|l|}{$>20$} \\
\hline Spain & 1 & 4 & 25 & $21 / 30^{\mathrm{a}}$ \\
\hline Total & 1 & - & - & $21 / 30$ \\
\hline \multicolumn{5}{|c|}{ Compound heterozygous mutations in RPE65 } \\
\hline Total & 22 & 22 & - & $90 / 187$ \\
\hline \multicolumn{5}{|l|}{0} \\
\hline Germany & 1 & 5 & 20 & $0 / 0$ \\
\hline Italy & 1 & 4 & 25 & $0 / 0$ \\
\hline Total & 2 & - & - & $0 / 0$ \\
\hline \multicolumn{5}{|l|}{$1-5$} \\
\hline Austria & 1 & 1 & 100 & $1 / 5$ \\
\hline Belgium & 1 & 1 & 100 & $1 / 5$ \\
\hline France & 2 & 2 & 100 & $2 / 10$ \\
\hline Germany & 2 & 5 & 40 & $2 / 10$ \\
\hline Italy & 3 & 4 & 75 & $3 / 15$ \\
\hline Portugal & 1 & 1 & 100 & $1 / 5$ \\
\hline Spain & 2 & 4 & 50 & $2 / 10$ \\
\hline Switzerland & 1 & 1 & 100 & $1 / 5$ \\
\hline The Netherlands & 2 & 3 & 67 & $2 / 10$ \\
\hline Total & 15 & - & - & $15 / 75$ \\
\hline \multicolumn{5}{|l|}{$11-20$} \\
\hline Germany & 1 & 5 & 20 & $11 / 20$ \\
\hline Total & 1 & - & - & $11 / 20$ \\
\hline \multicolumn{5}{|l|}{$>20^{10 t a l}$} \\
\hline Germany & 1 & 5 & 20 & $21 / 30^{\mathrm{a}}$ \\
\hline Spain & 1 & 4 & 25 & $21 / 30^{\mathrm{a}}$ \\
\hline The Netherlands & 1 & 3 & 33 & $21 / 30^{\mathrm{a}}$ \\
\hline Total & 3 & - & - & $63 / 90$ \\
\hline \multicolumn{5}{|l|}{ Do not know } \\
\hline Spain & 1 & 4 & 25 & $1 / 2^{b}$ \\
\hline Total & 1 & - & - & $1 / 2$ \\
\hline
\end{tabular}

The percentage of centers per country was calculated based on the total number of centers that replied for each country. $N$ refers to the number of centers. IRD, inherited retinal degeneration. ${ }^{\text {a }}$ Estimated number assuming 30 patients as maximum for centers with $>20$ patients. ${ }^{b}$ Estimated number assuming 2 patients as maximum for centers answering do not know. 
to only ask for estimated numbers in order to respect patient's confidentiality. It is important to recognize that not all variants in genes were disease-causing. An actual classification comprises at least 5 major types of mutations, that is, (1) pathogenic, (2) likely pathogenic, (3) uncertain significance, (4) likely benign, and (5) benign [20]. Likely pathogenic corresponds to class 4 and pathogenic to class 5 . Only class 4 and 5 mutations are considered to be clearly disease-causing, hence a genotype, where gene supplementation therapy with voretigene neparvovec (VN) is indicated [21]. Classification of missense mutations as pathogenic or likely pathogenic, that is, class 4 or 5 , can be challenging but is mandatory prior to treatment [20]. Previously unclassified mutations may be definitely identified as disease-causing with additional investigations such as segregation of the mutation in the family [22], or by testing the enzymatic activity of the mutant $[9,23]$. We, therefore, asked specifically for the estimated number of patients with biallelic class 4 and 5 mutations.

The identification of the EVICR.net member and name, function and contacts (e-mail and telephone) of the replier were requested as they are all EVICR.net members with a Confidentiality Disclosure Agreement in place.

\section{Statistical Analysis}

We conducted a descriptive analysis to all variables that was the same as recently reported for the general IRD survey [15]. Continuous variables were summarized using the following statistics: number $(n)$, mean, standard deviation (SD), median (P50), first and third quartiles (P25 and P75), minimum (Min), and maximum (Max). The frequency and percentages of observed levels were reported for all categorical measures. Statistical analyses were performed with Excel version 15.0.4433.1508 (Microsoft Office Home and Business 2013) and R version 3.6.0 (2019-04-26). We did not exclude questionnaires due to missing values. However, each analysis was restricted to repliers with no missing values for the respective question (i.e., total number of repliers differed between questions).

\section{Results}

Demographics of RPE65 Mutation-Associated IRDs in 22 EVICR.net Centers

The IRD survey was sent to 101 EVICR.net clinical research centers from 14 European countries and Israel [15]. Forty-nine percent of the 101 EVICR.net clinical research centers ( 49 centers) in 15 countries who had received the online survey responded. However, only 22 EVICR.net clinical centers have IRD patients with confirmed biallelic mutations in RPE65 identified in their centers (Fig. 1). The Netherlands reported the highest percentage of centers with patients with RPE65 mutationassociated IRDs, that is, $60 \%$, except for Austria with only 1 center as EVICR.net member (100\% of centers). Sixtyeight percent of the centers have only 1-5 IRD patients with confirmed homozygous mutations in RPE65 as well as only 1-5 IRD patients with confirmed compound het-
Table 3. Estimate of the number of IRD patients with confirmed biallelic mutations in RPE65 according to ACMG class 4 or 5

\begin{tabular}{lrrc}
\hline & $N$ & $\%$ & Min/max \\
\hline 0 & 2 & 9 & $0 / 0$ \\
$1-5$ & 11 & 50 & $11 / 55$ \\
$6-10$ & 0 & 0 & $0 / 0$ \\
$11-20$ & 0 & 0 & $0 / 0$ \\
$>20$ & 2 & 9 & $42 / 60^{\mathrm{a}}$ \\
Do not know & 7 & 32 & $7 / 14^{\mathrm{b}}$ \\
\hline Total & 22 & 100 & $60 / 129$ \\
\hline
\end{tabular}

Total of centers with RPE65

mutation-associated IRD patients $\quad 22 \quad 100 \quad-$

$N$ refers to the number of centers. IRD, inherited retinal degeneration. ${ }^{a}$ Estimated number assuming 30 patients as maximum for centers with $>20$ patients. ${ }^{\text {b}}$ Estimated number assuming 2 patients as maximum for centers answering do not know.

Table 4. Estimate of the number of IRD patients with confirmed biallelic mutations in RPE65 currently observed

\begin{tabular}{lrrc}
\hline & $N$ & $\%$ & Min/max \\
\hline 0 & 1 & 5 & $0 / 0$ \\
$1-5$ & 14 & 64 & $14 / 70$ \\
$6-10$ & 3 & 14 & $18 / 30$ \\
$11-20$ & 0 & 0 & $0 / 0$ \\
$>20$ & 3 & 14 & $63 / 90^{\mathrm{a}}$ \\
Do not know & 1 & 5 & $1 / 2^{\mathrm{b}}$ \\
\hline Total & 22 & 100 & $96 / 192$ \\
\hline Total of centers with RPE65 & & & \\
mutation-associated IRD patients & 22 & 100 & - \\
\hline
\end{tabular}

$N$ refers to the number of centers. IRD, inherited retinal degeneration. ${ }^{\text {a }}$ Estimated number assuming 30 patients as maximum for centers with $>20$ patients. ${ }^{b}$ Estimated number assuming 2 patients as maximum for centers answering do not know.

erozygous mutations in RPE65 (Table 1). Table 2 shows the number of centers with confirmed homozygous and compound heterozygous mutations in RPE65 per country and the estimated minimum and maximum number of patients.

The estimated number of IRD patients with confirmed biallelic mutations according to American College of Medical Genetics (ACMG) class 4 or 5 that have been identified per center was 0 in $9 \%$ of the centers, $1-5$ in 50 , and $>20$ in $9 \%$ of the centers (Table 3 ). The estimated number of IRD patients with confirmed biallelic 
Table 5. Frequency to recall the patients for follow-up by country

\begin{tabular}{|c|c|c|c|c|}
\hline Country & $N$ & $\%$ & $\begin{array}{l}\text { Total centers } \\
\text { at section } 5 \\
\text { per country }\end{array}$ & $\%$ per country \\
\hline \multicolumn{5}{|l|}{ Every 6 months } \\
\hline France & 1 & - & 2 & 50 \\
\hline Italy & 2 & - & 4 & 50 \\
\hline Portugal & 1 & - & 1 & 100 \\
\hline Spain & 1 & - & 4 & 25 \\
\hline Total & 5 & 23 & - & - \\
\hline \multicolumn{5}{|l|}{ Annually } \\
\hline Austria & 1 & - & 1 & 100 \\
\hline Belgium & 1 & - & 1 & 100 \\
\hline France & 1 & - & 2 & 50 \\
\hline Germany & 2 & - & 5 & 40 \\
\hline Italy & 2 & - & 4 & 50 \\
\hline Spain & 3 & - & 4 & 75 \\
\hline Switzerland & 1 & - & 1 & 100 \\
\hline The Netherlands & 1 & - & 3 & 33 \\
\hline Total & 12 & 55 & - & - \\
\hline \multicolumn{5}{|l|}{ Biennially } \\
\hline Germany & 2 & - & 5 & 40 \\
\hline The Netherlands & 2 & - & 3 & 67 \\
\hline Total & 4 & 18 & - & - \\
\hline \multicolumn{5}{|l|}{ Longer } \\
\hline Total & 0 & 0 & - & - \\
\hline \multicolumn{5}{|l|}{ Do not know } \\
\hline Total & 0 & 0 & - & - \\
\hline \multicolumn{5}{|c|}{ Other: age-dependent semiannually to biannually } \\
\hline Germany & 1 & - & 5 & 20 \\
\hline Total & 1 & 5 & - & - \\
\hline Total & 22 & 100 & - & - \\
\hline $\begin{array}{l}\text { Total of centers with RPE65 } \\
\text { mutation-associated IRD patients }\end{array}$ & 22 & 100 & - & - \\
\hline
\end{tabular}

The percentage of centers per country was calculated based on the total number of centers that replied for each country. $N$ refers to the number of centers. IRD, inherited retinal degeneration.

mutations currently followed per center is 0 in $5 \%$ of the centers, $1-5$ in $64,6-10$ in 14 , and $>20$ in $14 \%$ of the centers (Table 4 ). The mean number of patients per center was 6.6; however, $25 \%$ of the centers identified $>7$ patients (maximum $>20$ ). Tables 3 and 4 also indicate the estimated overall minimum and maximum number of patients.

From the identified RPE65 mutations, $82.8 \%$ were already reported mutations and $17.2 \%$ were novel mutations. Patients with biallelic RPE65 mutation-associated IRD had as referral diagnosis: early-onset severe retinal dystrophy (EOSRD) in 16.8\%, Leber congenital amaurosis (LCA) in $38.2 \%$, retinitis pigmentosa (RP)/rod-cone dystrophy in $28.1 \%$, and unclassified visual impairment in $17.0 \%$ of the cases (the mean per center). The mean percentage of centers that changed the referral diagnosis of RPE65 mutation-associated IRD patients was 30, and $25 \%$ of the centers changed the referral diagnosis in $>47.5 \%$ of the cases. Only $32 \%$ of the centers follow a specific referral process for RPE65 mutation-associated IRD patients. Online suppl. Table 1 shows the number of centers that follow a specific referral process per country.

\section{Follow-Up Visits}

Actual Practice

Fifty-five percent of the centers recall patients every year, 23\% every 6 months, and $18 \%$ every 2 years (Table 5). The frequency of follow-up visits for RPE65 mu- 
Table 6. Frequency that centers perform VA testing in RPE65 mutation-associated IRD patients

\begin{tabular}{lrc}
\hline & $N$ & $\%$ \\
\hline Monthly & 0 & 0 \\
Quarterly & 0 & 0 \\
Twice a year & 7 & 32 \\
Annually & 10 & 45 \\
Biennially & 4 & 18 \\
Longer & 0 & 0 \\
Other: with every visit & 1 & 5 \\
\hline Total & 22 & 100 \\
\hline
\end{tabular}

Total of centers with RPE65

mutation-associated IRD patients $\quad 22 \quad 100$

$N$ refers to the number of centers. IRD, inherited retinal degeneration; VA, visual acuity.

tation-associated IRD patients is only every 2 years in $40 \%$ of the centers in Germany and in $67 \%$ of the centers in the Netherlands (Table 5). On the other hand, the highest frequency of follow-up visits of every 6 months was reported in France, Italy, Portugal, and Spain (Table 5). Thirty-two percent of the centers replied that another institution also follows the RPE65 mutation-associated IRD patients.

\section{Previous Practice}

In the past, $59 \%$ of the centers saw patients every year, $14 \%$ every 6 months, $14 \%$ every 2 years, and 5\% longer (online suppl. Table 2). The time between visits for IRD patients in the past varied significantly in Germany and was mostly biennially in the Netherlands (online suppl. Table 2). On the other hand, the shortest mean time between visits in the past was every 6 months reported in Italy and Spain (online suppl. Table 2).

\section{Psychophysics}

Visual Acuity and Color Vision Testing

Visual acuity (VA) is tested in $45 \%$ of the 22 centers in RPE65 mutation-associated IRD patients every year, 32\% every 6 months, and $18 \%$ every 2 years (Table 6 ). The methods applied for VA testing in RPE65 mutation-associated IRD patients in the 22 centers are particularly ETDRS charts (59\%), Snellen charts (59\%), Number charts (41\%), and Lea Symbols ${ }^{\circledR}$ (32\%) (Table 7). The methods applied for color vision testing in RPE65 mutation-associated IRD patients in the centers are particularly Farnsworth Panel D15 (68\%) and Ishihara plates (55\%).
Table 7. Methods applied for VA testing in RPE65 mutationassociated IRD patients

\begin{tabular}{lrc}
\hline & $N$ & $\%$ \\
\hline ETDRS charts & 13 & 59 \\
Snellen charts & 13 & 59 \\
Number charts & 9 & 41 \\
Landolt rings & 4 & 18 \\
Tumbling "E" charts & 5 & 23 \\
BRVT & 2 & 9 \\
Teller acuity cards & 5 & 23 \\
Lea symbols & 7 & 32 \\
HOTV & 1 & 5 \\
\hline Total & 59 & $268^{\mathrm{a}}$ \\
\hline Total of centers with RPE65 & & \\
mutation-associated IRD patients & 22 & 100 \\
\hline
\end{tabular}

$N$ refers to the number of centers. IRD, inherited retinal degeneration; VA, visual acuity; BRVT, Berkeley rudimentary vision test. ${ }^{a}$ Multiple choices allowed.

Table 8. VF tests performed in RPE65 mutation-associated IRD patients

\begin{tabular}{lcc}
\hline & $N$ & $\%$ \\
\hline Static perimetry & 10 & 45 \\
Kinetic perimetry & 18 & 82 \\
Fundus-controlled perimetry & 4 & 18 \\
\hline Total & 32 & $145^{\mathrm{a}}$ \\
\hline Total of centers with RPE65 & & \\
mutation-associated IRD patients & 22 & 100 \\
\hline
\end{tabular}

$N$ refers to the number of centers. IRD, inherited retinal degeneration; VF, visual field. For detailed information of the specific devices used, see online suppl. Table 3a-e. ${ }^{a}$ Multiple choice allowed.

\section{Visual Field Testing}

Visual field (VF) testing results are listed in Table 8 and online suppl. Table 3a-e. Centers that manage RPE65 mutation-associated IRD patients mainly use kinetic perimetry $(82 \%)$ and static perimetry (45\%) (Table 8$)$. For static perimetry, all centers use Humphrey ${ }^{\circledR}$ (Carl Zeiss Meditec AG, Jena, Germany) (online suppl. Table 3a) and models of Octopus (Haag-Streit AG, Koeniz, Switzerland) used are shown in online suppl. Table $3 \mathrm{~b}$. Of the centers, $83 \%$ use Goldmann (manual) for kinetic perimetry (online suppl. Table 3c) and the models of Octopus (Haag-Streit AG, Koeniz, Switzerland) used are shown in 
Table 9. Number of VF tests (static or kinetic) performed per each RPE65 mutation-associated IRD patient

\begin{tabular}{|c|c|c|c|c|}
\hline & \multicolumn{2}{|c|}{ Static } & \multicolumn{2}{|c|}{ Kinetic } \\
\hline & $n$ & $\%$ & $n$ & $\%$ \\
\hline$<5$ & 15 & 68 & 14 & 64 \\
\hline $5-10$ & 4 & 18 & 5 & 23 \\
\hline $11-20$ & 1 & 5 & 2 & 9 \\
\hline$>20$ & 0 & 0 & 0 & 0 \\
\hline Do not know & 2 & 9 & 1 & 5 \\
\hline Total & 22 & 100 & 22 & 100 \\
\hline $\begin{array}{l}\text { Total of centers with RPE65 } \\
\text { mutation-associated IRD patients }\end{array}$ & 22 & 100 & 22 & 100 \\
\hline
\end{tabular}

$n$ refers to the number of centers. IRD, inherited retinal degeneration; VF, visual field.
Table 10. Centers performing FST in RPE65 mutation-associated IRD patients

\begin{tabular}{lrr}
\hline & $N$ & $\%$ \\
\hline Yes & 8 & 36 \\
Blue, red, white testing & 6 & - \\
White testing only & 2 & - \\
No & 14 & 64 \\
\hline Total & 22 & 100 \\
\hline Total of centers with RPE65 & & \\
mutation-associated IRD patients & 22 & 100 \\
\hline
\end{tabular}

$N$ refers to the number of centers. FST, full-field stimulus testing; IRD, inherited retinal degeneration.

online suppl. Table 3d. For fundus-controlled perimetry, $75 \%$ of the centers use MP3 (NIDEK Co. Ltd., Aichi, Japan) (online suppl. Table 3e).

The number of VF tests (static) that each center performs per each RPE65 mutation-associated IRD patient was $<5$ in $68 \%$ of the centers, $5-10$ in 18, and $11-20$ in $5 \%$ (Table 9). The number of VF tests (kinetic) that each center performs per each RPE65 mutation-associated IRD patient was $<5$ in $64 \%$ of the centers, $5-10$ in 23 , and $11-$ 20 in $9 \%$ (Table 9).

\section{Two-Color-Threshold Perimetry and Full-Field}

\section{Stimulus Threshold}

Only 9\% of the 22 centers that manage RPE65 mutation-associated IRD patients perform Two-ColorThreshold perimetry (2 CT-perimetry), and 36\% of the 22 centers perform Full-Field Stimulus Testing (FST) in IRD patients (Table 10). From those centers, $75 \%$ performs red, blue, and white testing (Table 10). Online suppl. Table 4 shows the devices used in RPE65 mutation-associated IRD patients.

\section{Pupillometry and Mobility Testing at Defined Light Levels}

Only $14 \%$ of the 22 centers that manage RPE65 mutation-associated IRD patients perform pupillometry. From these 3 centers, 2 (67\%) perform chromatic pupillometry [24-26]. Only $5 \%$ of the 22 centers that manage RPE65 mutation-associated IRD patients perform mobility testing at defined light levels (Ora-VNC ${ }^{\mathrm{TM}}$, Ora, Inc., Andover, MA, USA).

\section{Retinal Imaging and Fundus Autofluorescence \\ Recording}

Fifty percent of the centers perform fundus imaging in RPE65 mutation-associated IRD patients every year, $27 \%$ every 6 months, and $18 \%$ every 2 years (Table 11 ). Fundus autofluorescence (FAF) recording is performed every year in $50 \%$ of their RPE65 mutation-associated IRD patients, every 6 months in 18\%, every 2 years in 14\%, and at even longer intervals in 9\% (Table 11). Spectral domain-optical coherence tomography (SD-OCT) is performed every year in 50\% of the 22 centers in RPE65 mutation-associated IRD patients, every 6 months in $27 \%$, and every 2 years in $18 \%$ (Table 11 ).

\section{Discussion}

This is the first comprehensive survey on diagnosis and management of RPE65 mutation-associated IRDs among all EVICR.net centers in Europe and Israel. We 
Table 11. Frequency that centers perform fundus imaging, FAF and SD-OCT recording in RPE65 mutation-associated IRD patients

\begin{tabular}{|c|c|c|c|c|c|c|}
\hline & \multicolumn{2}{|c|}{ Fundus imaging } & \multicolumn{2}{|c|}{ FAF } & \multicolumn{2}{|c|}{ SD-OCT } \\
\hline & $n$ & $\%$ & $n$ & $\%$ & $n$ & $\%$ \\
\hline Monthly & 0 & 0 & 0 & 0 & 0 & 0 \\
\hline Quarterly & 0 & 0 & 0 & 0 & 0 & 0 \\
\hline Twice a year & 6 & 27 & 4 & 18 & 6 & 27 \\
\hline Annually & 11 & 50 & 11 & 50 & 11 & 50 \\
\hline Biennially & 4 & 18 & 3 & 14 & 4 & 18 \\
\hline Longer & 0 & 0 & 2 & 9 & 0 & 0 \\
\hline Other: with every visit & 1 & 5 & 1 & 5 & 1 & 5 \\
\hline $\begin{array}{l}\text { Other: we try to retrieve FAF images on the first visits, } \\
\text { but if we do not succeed, and know that is not a hypomorph mutation, } \\
\text { we do not repeat it on every follow-up visit }\end{array}$ & 0 & 0 & 1 & 5 & 0 & 0 \\
\hline Total & 22 & 100 & 22 & 100 & 22 & 100 \\
\hline Total of centers with RPE65 mutation-associated IRD patients & 22 & 100 & 22 & 100 & 22 & 100 \\
\hline
\end{tabular}

$n$ refers to the number of centers. IRD, inherited retinal degeneration; FAF, fundus autofluorescence; SD-OCT, spectral domainoptical coherence tomography.

are not aware of a similar survey in other parts of the world. Only 22 of the 49 responding centers follow RPE65 mutation-associated IRD patients (Fig. 1).

Adding the estimated numbers in the 22 EVICR.net centers, the range of patients with homozygous mutations in RPE65 goes from 65 to 155 and the range of patients with compound heterozygous mutations in RPE65 goes from 90 to 187 . Only 60 to 129 patients were estimated to carry class 4 and 5 mutations (Tables $1,3,4$ ). So, a conservative estimation of patients eligible to gene augmentation therapy would be at least 60 , and 129 at max. As we do not know the age or the current visual function of the estimated number of patients with class 4 and 5 mutations, the actual number is probably even lower. Given the estimated overall number of patients with biallelic mutations in Europe, that is, 2,500 patients based on a prevalence of $1: 300,000[1,18]$, we can confirm an unmet need of patient identification. The fact that $17 \%$ of the cases were diagnosed as unclassified visual impairment and $28.1 \%$ with RP indicates that a significant number of patients may go undiagnosed if not referred to a specialized center. Without molecular genetic diagnostics, $45.1 \%$ of the patients are at risk not to be associated with the RPE65 genotype. This is unfortunate, as costeffectiveness of the gene augmentation therapy with $\mathrm{VN}$ has been shown by several groups, not to speak about the alleviation of the burden of a disease that left untreated usually ends in blindness by the end of the third to fourth decade of life $[8,12,19]$. To identify all patients eligible for gene therapy with $\mathrm{VN}$, patients with the diagnosis $\mathrm{RP} /$ rod-cone dystrophy and unclassified visual impairment summarizing to $45.1 \%$ are of particular interest. They might have received their diagnosis well before the advent of gene therapy, and therefore reevaluation and genetic testing should be considered in this patient group. Another reason could be that ophthalmologists, pediatricians, neurologists, or general practitioners are not yet aware of the availability of $\mathrm{VN}$ therapy. To reach those patients and their physicians, disease awareness and educational campaigns in scientific journals and conferences, patients' organizations meetings, and even in the public media might be useful. To detect early stages, it could be discussed to include testing of RPE65 in the already established neonatal screening program for severe genetic diseases amenable to therapy.

Besides the established treatment with VN, improvements are sought by novel gene augmentation therapies explored in human induced pluripotent stem cell-derived retinal pigment epithelial cells $[27,28]$, and by gene correction via CRISPR-Cas9 [29]. At present, both developments are in the preclinical phase. To test such novel approaches, there is a need to identify more patients with the RPE65 genotype, who would be interested to take part in further clinical trials.

\section{Psychophysics}

The survey asked for the work-up and follow-up of patients with suspected or confirmed biallelic mutations 
in RPE65. All centers perform a comprehensive eye examination (Tables $6-10$, online suppl. Tables 3,4 ). The results concerning methods used for VA testing reflect the fact that young patients are less frequently seen in the 22 centers that do follow such patients (online suppl. Table 2). The most frequently used tests are ETDRS and Snellen charts, each at 59\% (Table 7). As multiple answers were allowed, it is not possible to conclude about the absolute frequency of the use of ETDRS charts. Dong et al. [30] reported in 2003 that ETDRS charts were used in 16/19 studies since the publication of the charts in 1982. Kaiser compared the validity of ETDRS versus Snellen in his AOI thesis [31]. The validity of ETDRS charts was also tested in children [32]. Recently, repeatability and agreement of VA testing using the ETDRS Number chart, Landolt C chart, or ETDRS Alphabet chart in eyes with or without sight-threatening diseases was reported [33]. This is of interest for the PERCEIVE Registry, a post-authorization observational safety study for patients treated with VN, sponsored by Novartis (ENCePP CLTW888A12401, http://www.encepp.eu/encepp/viewResource.htm?id=37005). If all centers treating patients with VN use ETDRS charts, the scientific value of the VA data will be high. LEA symbols are also available in the format used in the ETDRS charts. Interpretation of VA data should acknowledge that repeated measurements even the same day can vary significantly in patients with RPE65 mutations [34].

Concerning VF testing, kinetic perimetry is done in most centers, mostly Goldmann perimetry (Table 8 and online suppl. Table 3c). Static perimetry is used in about half of the centers (Table 8 and online suppl. Table 3a). Fundus-controlled perimetry is only used in 4 centers (18\%). As the PERCEIVE Registry does not require specific methods for VF testing, it is likely that quantitative data on the long-term effect of VN collected in all participating centers will be limited to kinetic perimetry. Unfortunately, VF data obtained with kinetic perimetry have to be interpreted with caution [35-38]. FDA encourages sponsors to explore a wide spectrum of potential clinical end points and other clinical effects in early-phase trials, such as retinal imaging, VA (low and high luminance), VFs, color vision, contrast sensitivity, and functional vision (i.e., how well the patient performs vision-related activities of daily living). For later phase trials, primary efficacy end points should reflect clinical benefit, such as improvement in function or symptoms (https://www. fda.gov/media/124641/download). Therefore, additional readout parameters were included in the phase 1-3 studies on RPE65 gene therapy with $\mathrm{VN}$ such as the specifi- cally developed multi-luminance mobility test (MLMT) [39]. The only spatially resolved VF testing separating the rod from the cone pathway is 2 CT-perimetry [26]. This is interesting as all data published so far on the effects of $\mathrm{VN}$ therapy indicate a clear effect on the rod function, but an ambiguous effect on the cone function $[13,40]$. Unfortunately, this method is only used in $9 \%$ of the 22 EVICR. net centers that do manage RPE65 patients as 2 CT-perimetry is not a commercially available device.

A psychophysical test that globally differentiates between the rod and cone pathway is chromatic FST $[19,26$, 41]. In the 22 EVICR.net centers that follow RPE65 patients, chromatic FST is used in only $6 / 8$ centers that use FST (Table 10 and online suppl. Table 4). Ganzfeld electroretinogram (ERG) is an objective test that separates the global rod from the global cone function in the retina. Unfortunately, in the majority of patients with biallelic mutations in RPE65, rod and cone responses are not measurable at the time of diagnosis. The natural history study on 70 patients with RPE65 mutation-associated IRDs found 98 full-field-ERGs on 60 patients. The scotopic ERG responses were extinguished in $78.6 \%$, and the photopic, that is, cone-mediated responses in $61.2 \%$. The mean age of patients with residual rod- and cone-mediated responses was 10.5 and 9.8 years [8, 9, 39].

Another test able to separate the rod from the cone function is chromatic pupillometry [26]. Only $3 / 22$ EVICR.net centers that follow RPE65 mutation-associated IRD patients perform pupillometry (14\%), and only $2 / 3(66 \%)$ perform chromatic pupillometry. The test that was decisive for the approval of $\mathrm{VN}$ for the treatment of biallelic RPE65 mutation-associated IRDs was the MLMT, as a highly patient relevant readout parameter, and considered to be less variable than VA and VF results [42]. Only 5\% of the 22 EVICR.net centers that follow RPE65 patients have a mobility course, as it is expensive and time consuming. This is unfortunate as mobility is important for the quality of life, and hence one of the possible major benefits of VN therapy in patients with RPE65 mutationassociated IRDs. Therefore, tests separating rod from cone-mediated vision including mobility testing may not yield statistically meaningful results in the PERCEIVE Registry due to limited data availability.

\section{Retinal Imaging}

Retinal fundus imaging including FAF recording is performed in all 22 EVICR.net centers following patients with RPE65 mutation-associated IRDs (Table 11). Lack of FAF has been reported as a hallmark sign of the RPE65 phenotype [9]. In patients with hypomorphic mutations, 
some FAF may be present or develop over the years [29, Lorenz et al., unpublished data]. A recent study using quantitative FAF has shown that FAF may become noticeable after VN therapy[43]. As some centers reported to register FAF images only once when it is not measurable, it is now highly recommended to systematically take FAF images after VN therapy. SD-OCT is regularly tested in all EVICR.net centers following patients with RPE65 mutation related IRDs. Half of the centers perform SDOCT at an annual basis, and about a quarter twice every year (Table 11). SD-OCT is an important readout parameter, as has been shown again recently [44]. Following treatment, it should also be an obligatory test to monitor the disease course as it has been shown that degeneration may continue in the area treated [16], depending on the percentage of photoreceptors preserved at the time of treatment [17].

\section{Frequency of Follow-Up}

Half of the 22 EVICR.net centers that follow patients with RPE65 related IRDs see their patients annually, 27\% twice a year or $18 \%$ biannually (Tables 5, 6, 9, 11 and online suppl. Table 2). Usually, all tests used for phenotyping are repeated at each visit. Given the validity of the tests, highly significant data on the natural course of the disease are available in all 22 centers that follow RPE65 patients. Depending on the number of patients eligible to therapy, for example, according to the criteria published by the professional ophthalmological associations in Germany [21], we can expect a significant number of patients treated with $\mathrm{VN}$ in the future, and followed adequately.

Annual follow-up appears a reasonable compromise to monitor the natural course. Following subretinal gene therapy, more frequent follow-up examinations, for example., 1, 3, 6 months, and 1 year appear appropriate during the 1st year post therapy to monitor any changes related to the therapy. Of note, in order to develop recommendations as to the management of RPE65 mutationassociated IRDs, and IRDs in general, it is also important to take into consideration age of onset, duration of the disease, and severity of the mutations.

\section{Weaknesses of the Survey}

To estimate the overall coverage of eye departments caring for patients with IRDs, and in particular with RPE65 mutation-associated IRDs, we have to acknowledge that the number of EVICR.net centers responding to the survey is significantly smaller than the overall number of eye departments and in addition varies among countries. For example, in Belgium there are at present 3
EVICR.net centers but 5 university hospitals. In Germany, $15 / 38$ university departments and 2/62 non-university eye departments are EVICR.net members. Only $5 \mathrm{Ger}-$ man EVICR.net centers of the 10 repliers in Germany have IRD patients with confirmed biallelic mutations in RPE65. In the Netherlands, there are 8 University Eye Departments and 1 non-university Eye Hospital; 5/9 are EVICR-net centers, and 3/5 replied to the survey. In addition, patients are also seen outside eye departments (in private practice) and not necessarily referred back to eye departments for more precise classification once they have the diagnosis of RP or central visual impairment. For example, in Germany, the overall number of practicing ophthalmologists is about 6,500 , but about two-thirds work outside eye departments. Therefore, it is difficult to give a precise estimation of the overall number of patients with RPE65 mutation-associated IRDs in Europe. Another way to estimate the number of patients with RPE65 mutation-associated IRDs covered by our survey is to compare the number of patients estimated in the EVICR. net centers in each country to the estimated overall prevalence. In Germany, 45 to at least 57 RPE65 mutationassociated IRD patients are followed in 5 German EVICR. net centers. With a population of about 83 million and given the estimated prevalence of IRD cases of 1:3,000 and an estimated prevalence of RPE65 mutation-associated IRD patients of $1-2 \%$ [18], the overall number of patients with RPE65 mutations in Germany would be $277-553$. This would mean $8-20 \%$ of all RPE65 mutationassociated IRD patients in Germany are followed in the 5 German EVICR.net centers. In the Netherlands, due to collaboration within the RD5000 study group, precise numbers are available in Rotterdam where currently 46 patients with RPE65 mutation-associated IRDs are seen. Fifty percent are from a genetic isolate [12]. Some more patients are known outside the patient cohort seen in Rotterdam, so the overall estimated prevalence is in the order of 65-75 patients for a population of 17.3 million. In Portugal, for a population of $\sim 10$ million, estimates anticipate an overall number of RPE65 mutation-associated IRD patients between 33 and 67 . The numbers that resulted from this survey indicate a considerably smaller number. Two possible reasons are (1) patients being followed at other centers that are currently not EVICR.net members and/or (2) patients that remain unidentified because genetic testing is not routinely performed (nor available) in all centers.

Taking the numbers from Germany, the Netherlands, and Portugal together, the estimated overall prevalence is quite similar, but differences are evident with regard to 
the percentage of already identified patients. The Netherlands has the highest number of already identified patients with biallelic mutations in RPE65.

Although done on purpose, the fact that we only asked for estimated numbers of patients followed in the EVICR. net centers, can further influence the real number of patients with RPE65 mutation-associated IRDs. In addition, we do not know how many patients are seen in several centers in parallel. Of note, only 14/47 European countries (including Russia and Turkey) participated in the survey. Altogether, we cannot conclude with sufficient precision on the number of patients with RPE65 mutation-associated IRDs in Europe.

In the survey, we asked about the estimated number of patients with class 4 and 5 mutations (online suppl. material - online questionnaire). In the instruction part, we did not elaborate on what class 4 and 5 mutations mean. Although the classification system has been published [20], some responders may not have been familiar with this classification system. As at present only class 4 and class 5 mutations are considered to be clearly diseasecausing and hence suited for VN therapy, at least according to the recommendations of the German Ophthalmology Societies [21], it is important to identify such patients.

\section{Strengths of the Survey}

This survey for the first time provides estimates on the number of patients with RPE65 mutation-associated IRDs, followed in 22 EVICR.net centers. As all EVICR. net centers are certified and follow the same standard operating procedures, it can be expected that the data available have been collected and archived in a comparable way. The survey has yielded precise data on the test methods and devices used. Of note, the number of centers that use more sophisticated tests such as the FST, pupillometry, fundus-controlled perimetry, and mobility course is still limited. The PERCEIVE Registry, sponsored by Novartis, aims to observe and understand the clinical impact of VN in a real-world setting. Through systematic collection of data on adverse events (AE and SAE), the study will characterize the long-term safety profile of $\mathrm{VN}$ over a period of 5 years (ENCePP CLTW888A12401, http:// www.encepp.eu/encepp/viewResource.htm?id=37005, overall duration 10 years). In addition, basic data such as $\mathrm{VA}, \mathrm{VF}$, and OCT are collected by the centers on a voluntary basis to monitor long-term functional and morphological outcome. When available, more sophisticated data such as FST and eventually MLMT will also be entered, but as they are not part of the standard of care, they may not be conducted on the majority of patients. Given the significant socioeconomic burden to the society, it would be of great interest to collect such additional data. They can quantitatively document objective treatment effects relevant to patient's quality of life and in more detail than the patient-reported outcomes questionnaires that are currently part of the PERCEIVE Registry. Efforts should be made in this direction.

\section{Conclusion}

This survey on diagnosis and management of RPE65 mutation-associated IRDs has provided important information on the actual situation in the 22 EVICR.net centers that have answered and followed such patients. The EVICR.net was a unique platform for collecting the data. These baseline data, previously not explored on such a scale, are of great importance to policy makers, clinicians, patient advocate groups, researchers, and others to inform and improve bottlenecks in the provision of optimal care for patients with RPE65 mutation-associated IRDs. Recommendations as to future steps include suggestions as to the timely detection of as many patients as possible who might benefit from $\mathrm{VN}$ therapy and for follow-up studies. The latter is important in view of cost-effectiveness and patient satisfaction of VN therapy. Guidelines on the diagnosis and management of RPE65 mutationassociated IRDs in particular and on IRDs in general can be developed based on the results of this unique data set.

\section{Acknowledgements}

We thank Dr. Sue Lacey, PhD (former Novartis employee), for providing valuable comments in the design and content of the IRD questionnaire, as well as to the results report. We thank Dr. Isabelle Audo, MD PhD (CHNO of Quinze-Vingts, Paris, France), for reviewing the first draft of the survey. We also thank Markus Preising, $\mathrm{PhD}$ (Dept. of Ophthalmology, Justus-Liebig-University Giessen, Germany), who helped with the format and contents of the first draft of the survey. We thank the Novartis team for the final review of this manuscript and for providing valuable scientific and editorial comments.

\section{Statement of Ethics}

This survey was reviewed and approved by the AIBILI Ethics Committee - Comissão de Ética para a Saúde, prior to its dissemination to the 101 EVICR.net clinical centers members and was in accordance with the World Medical Association Declaration of Helsinki. As no personal data were collected, the use of a written and informed consent form was not applicable. 


\section{Conflict of Interest Statement}

The authors have no conflicts of interest to declare.

\section{Funding Sources}

Novartis Pharma AG through a Scientific Collaboration Agreement between Novartis and AIBILI, the Coordinating Center of EVICR.net funded this study. Novartis provided nonbinding comment/input to the IRD Survey Expert Committee into all elements of the scientific collaboration agreement.

\section{Author Contributions}

The IRD questionnaire was designed by B.L., J.T., L.I.B., J.P.M., and H.P.N.S.; J.T. and B.L. analyzed data. B.L. and J.T. wrote the manuscript. L.I.B, J.P.M., and H.P.N.S. reviewed and complemented the manuscript. B.L., J.T., L.I.B., J.P.M., and H.P.N.S. approved the final manuscript.

\section{References}

1 Galvin O, Chi G, Brady L, Hippert C, Del Valle Rubido M, Daly A, et al. The Impact of Inherited Retinal Diseases in the Republic of Ireland (ROI) and the United Kingdom (UK) from a cost-of-illness perspective. Clin Ophthalmol. 2020 Mar; 14:707-19.

2 Johnson S, Buessing M, O'Connell T, Pitluck $S$, Ciulla TA. Cost-effectiveness of voretigene neparvovec-rzyl vs standard care for RPE65mediated inherited retinal disease. JAMA Ophthalmol. 2019 Oct;137(10):1115.

3 Uhrmann MF, Lorenz B, Gissel C. Cost effectiveness of voretigene neparvovec for RPE65mediated inherited retinal degeneration in Germany. Transl Vis Sci Technol. 2020 Aug; 9(9): 17.

4 Viriato D, Bennett N, Sidhu R, Hancock E, Lomax H, Trueman D, et al. An economic evaluation of voretigene neparvovec for the treatment of biallelic RPE65-mediated inherited retinal dystrophies in the UK. Adv Ther. 2020 Mar;37(3):1233-47.

5 Zimmermann M, Lubinga SJ, Banken R, Rind D, Cramer G, Synnott PG, et al. Cost utility of voretigene neparvovec for biallelic RPE65mediated inherited retinal disease. Value Health. 2019 Feb;22(2):161-7.

6 Marlhens F, Bareil C, Griffoin JM, Zrenner E, Amalric P, Eliaou C, et al. Mutations in RPE65 cause Leber's congenital amaurosis. Nat Genet. 1997 Oct;17(2):139-41.

$7 \mathrm{Gu}$ SM, Thompson DA, Srikumari CR, Lorenz B, Finckh U, Nicoletti A, et al. Mutations in RPE65 cause autosomal recessive childhood-onset severe retinal dystrophy. Nat Genet. 1997 Oct;17(2):194-7.

8 Chung DC, Bertelsen M, Lorenz B, Pennesi ME, Leroy BP, Hamel CP, et al. The natural history of inherited retinal dystrophy due to biallelic mutations in the RPE65 gene. Am J Ophthalmol. 2019 Mar;199:58-70.

9 Lorenz B, Poliakov E, Schambeck M, Friedburg C, Preising MN, Redmond TM. A comprehensive clinical and biochemical functional study of a novel RPE65 hypomorphic mutation. Invest Ophthalmol Vis Sci. 2008 Dec; 49(12):5235-42.
10 Hull S, Holder GE, Robson AG, Mukherjee R, Michaelides M, Webster AR, et al. Preserved visual function in retinal dystrophy due to hypomorphic RPE65 mutations. Br J Ophthalmol. 2016 Nov; 100(11):1499-505.

11 Kumaran N, Rubin GS, Kalitzeos A, Fujinami $\mathrm{K}$, Bainbridge JWB, Weleber RG, et al. A cross-sectional and longitudinal study of retinal sensitivity in RPE65-associated Leber congenital amaurosis. Invest Ophthalmol Vis Sci. 2018 Jul;59(8):3330.

12 Pierrache LHM, Ghafaryasl B, Khan MI, Yzer S, van Genderen MM, Schuil J, et al. Longitudinal study of RPE65-associated inherited retinal degenerations. Retina. 2020 Sep;40(9): 1812-28.

13 Russell S, Bennett J, Wellman JA, Chung DC, Yu Z-F, Tillman A, et al. Efficacy and safety of voretigene neparvovec (AAV2-hRPE65v2) in patients with RPE65-mediated inherited retinal dystrophy: a randomised, controlled, open-label, phase 3 trial. Lancet. 2017 Aug; 390(10097):849-60.

14 Bennett J, Wellman J, Marshall KA, McCague S, Ashtari M, DiStefano-Pappas J, et al. Safety and durability of effect of contralateral-eye administration of AAV2 gene therapy in patients with childhood-onset blindness caused by RPE65 mutations: a follow-on phase 1 trial. Lancet. 2016 Aug;388(10045):661-72.

15 Lorenz B, Tavares J, van den Born LI, Marques JP, Scholl HPN. Current management of Inherited Retinal Degenerations (IRD) patients in Europe. Results of a multinational survey by the European Vision Institute Clinical Research Network EVICR.net. Ophthalmic Res. 2021 Jan. Epub ahead of print.

16 Cideciyan AV, Jacobson SG, Beltran WA, Sumaroka A, Swider M, Iwabe S, et al. Human retinal gene therapy for Leber congenital amaurosis shows advancing retinal degeneration despite enduring visual improvement. Proc Natl Acad Sci U S A. 2013 Feb;110(6):E51725.
17 Gardiner KL, Cideciyan AV, Swider M, Dufour VL, Sumaroka A, Komáromy AM, et al. Long-term structural outcomes of late-stage RPE65 gene therapy. Mol Ther. 2020 Jan; 28(1):266-78.

18 Pontikos N, Arno G, Jurkute N, Schiff E, BaAbbad R, Malka S, et al. Genetic basis of inherited retinal disease in a molecularly characterized cohort of more than 3,000 families from the United Kingdom. Ophthalmology. 2020 Oct;127(10):1384-94.

19 Roman AJ, Schwartz SB, Aleman TS, Cideciyan AV, Chico JD, Windsor EA, et al. Quantifying rod photoreceptor-mediated vision in retinal degenerations: dark-adapted thresholds as outcome measures. Exp Eye Res. 2005 Feb;80(2):259-72.

20 Richards S, Aziz N, Bale S, Bick D, Das S, Gastier-Foster J, et al. Standards and guidelines for the interpretation of sequence variants: a joint consensus recommendation of the American College of Medical Genetics and Genomics and the Association for Molecular Pathology. Genet Med. 2015 May; 17(5):405-24.

21 German Society of Ophthalmology (Deutsche Ophthalmologische Gesellschaft, DOG); German Retina Society e. V. (Retinologische Gesellschaft e. V., RG); Professional Association of German Ophthalmologists (Berufsverband der Augenärzte Deutschlands e. V., B). Statement of the DOG, the RG, and the BVA on the therapeutic use of voretigene neparvovec (LuxturnaTM) in ophthalmology. English version. Der Ophthalmol. 2020 Jan;117(Suppl 1):16-24.

22 Motta F, Martin R, Porto F, Wohler E, Resende R, Gomes $\mathrm{C}$, et al. Pathogenicity reclassification of RPE65 missense variants related to leber congenital amaurosis and early-onset retinal dystrophy. Genes. 2019 Dec;11(1):24

23 Yang U, Gentleman S, Gai X, Gorin MB, Borchert MS, Lee TC, et al. Utility of in vitro mutagenesis of RPE65 protein for verification of mutational pathogenicity before gene therapy. JAMA Ophthalmol. 2019 Dec;137(12): 1381 
24 Kardon R, Anderson SC, Damarjian TG, Grace EM, Stone E, Kawasaki A. Chromatic pupillometry in patients with retinitis pigmentosa. Ophthalmology. 2011 Feb;118(2): 376-81.

25 Park JC, Moura AL, Raza AS, Rhee DW, Kardon RH, Hood DC. Toward a clinical protocol for assessing rod, cone, and melanopsin contributions to the human pupil response. Invest Ophthalmol Vis Sci. 2011 Aug;52(9): 6624-35.

26 Lorenz B, Strohmayr E, Zahn S, Friedburg C, Kramer M, Preising M, et al. Chromatic pupillometry dissects function of the three different light-sensitive retinal cell populations in RPE65 deficiency. Invest Ophthalmol Vis Sci. 2012 Aug;53(9):5641-52.

27 Georgiadis A, Duran Y, Ribeiro J, AbelleiraHervas L, Robbie SJ, Sünkel-Laing B, et al. Development of an optimized AAV2/5 gene therapy vector for Leber congenital amaurosis owing to defects in RPE65. Gene Ther. 2016 Dec;23(12):857-62.

28 Georgiadis A, Duran Y, Ribeiro J, AbelleiraHervas L, Robbie SJ, Sünkel-Laing B, et al. Correction: development of an optimized AAV2/5 gene therapy vector for Leber congenital amaurosis owing to defects in RPE65. Gene Ther. 2018 Sep;25(6):450.

29 Jo DH, Song DW, Cho CS, Kim UG, Lee KJ, Lee $\mathrm{K}$, et al. CRISPR-Cas9-mediated therapeutic editing of Rpe65 ameliorates the disease phenotypes in a mouse model of Leber congenital amaurosis. Sci Adv. 2019 Oct; 5(10):eaax1210.

30 Dong LM, Marsh MJ, Hawkins BS. Measurement and analysis of visual acuity in multicenter randomized clinical trials in the United States: findings from a survey. Ophthalmic Epidemiol. 2003 Jan;10(3):149-65.
31 Kaiser PK. Prospective evaluation of visual acuity assessment: a comparison of snellen versus ETDRS charts in clinical practice (An AOS Thesis). Trans Am Ophthalmol Soc. 2009 Dec;107:311-24.

32 Manny RE, Hussein M, Gwiazda J, MarshTootle W. Repeatability of ETDRS visual acuity in children. Invest Ophthalmol Vis Sci. 2003 Aug;44(8):3294.

33 Chaikitmongkol V, Nanegrungsunk O, Patikulsila D, Ruamviboonsuk P, Bressler NM. Repeatability and agreement of visual acuity using the ETDRS number chart, Landolt C chart, or ETDRS alphabet chart in eyes with or without sight-threatening diseases. JAMA Ophthalmol. 2018 Mar;136(3):286.

34 Cideciyan AV, Swider M, Aleman TS, Feuer WJ, Schwartz SB, Russell RC, et al. Macular function in macular degenerations: repeatability of microperimetry as a potential outcome measure for ABCA4 -associated retinopathy trials. Invest Ophthalmol Vis Sci. 2012 Feb;53(2):841.

35 Vonthein R, Rauscher S, Paetzold J, Nowomiejska K, Krapp E, Hermann A, et al. The normal age-corrected and reaction time-corrected isopter derived by semi-automated kinetic perimetry. Ophthalmology. 2007 Jun;114(6): 1065-72.e2.

36 Nowomiejska K, Brzozowska A, Zarnowski T, Rejdak R, Weleber RG, Schiefer U. Variability in isopter position and fatigue during semiautomated kinetic perimetry. Ophthalmologica. 2012;227(3):166-72.

37 Rowe FJ, Czanner G, Somerville T, Sood I, Sood D. Octopus 900 automated kinetic perimetry versus standard automated static perimetry in glaucoma practice. Curr Eye Res. 2021 Jan;46(1):83-95.
38 Zrenner E, Holder GE, Schiefer U, Wild JM. Quality control procedures and baseline values for electroretinography, perimetry, color vision, and visual acuity in an international multicenter study: observations from a safety trial in chronic stable angina pectoris. Trans Vis Sci Technol. 2020 Jul;9(8):38.

39 Paunescu K, Wabbels B, Preising MN, Lorenz $B$. Longitudinal and cross-sectional study of patients with early-onset severe retinal dystrophy associated with RPE65 mutations. Graefes Arch Clin Exp Ophthalmol. 2005 May;243(5):417-26.

40 Maguire AM, Russell S, Wellman JA, Chung DC, Yu ZF, Tillman A, et al. Efficacy, safety, and durability of voretigene neparvovec-rzyl in RPE65 mutation-associated inherited retinal dystrophy: results of phase 1 and 3 trials Ophthalmology. 2019 Sep;126(9):1273-85.

41 Jacobson SG, Aleman TS, Cideciyan AV, Roman AJ, Sumaroka A, Windsor EA, et al. Defining the residual vision in Leber congenital amaurosis caused by RPE65 mutations. Invest Ophthalmol Vis Sci. 2009 May;50(5):2368.

42 Chung DC, McCague S, Yu ZF, Thill S, DiStefano-Pappas J, Bennett J, et al. Novel mobility test to assess functional vision in patients with inherited retinal dystrophies. Clin Exp Ophthalmol. 2018 Apr;46(3):247-59.

43 Levi SR, Oh JK, de Carvalho JRL, Mahajan VB, Tsang SH, Sparrow JR. Quantitative autofluorescence following gene therapy with voretigene neparvovec. JAMA Ophthalmol. 2020 Aug;138(8):919.

44 Kumaran N, Georgiou M, Bainbridge JWB, Bertelsen M, Larsen M, Blanco-Kelly F, et al. Retinal structure inRPE65-associated retinal dystrophy. Invest Ophthalmol Vis Sci. 2020 Apr;61(4):47. 